\title{
FINITE ELEMENT MODELING PIEZOELECTRIC MATERIALS UNDER THERMAL LOADS WITH APPLICATION FOR QUANTUM DOTS
}

Significant research has been done in the analysis of properties of quantum dots over the previous decade. A 3D finite element model is developed to analyze quantum dots $(Q D)$ under static thermal loads. The lattice mismatch between the quantum dot and the piezoelectric matrix is created by different thermal properties of materials at enhanced temperature of this electronic structure. The fully coupled thermo-piezoelectricity is applied to the analysis of the problem. Commercial FEM software ANSYS was used for analysis. Finite element numerical results are given for the dot with a cubic shape. Numerical results for the InAs/GaAs QD nanostructure show that the elastic and electric fields are strongly influenced by the differences between the material properties of the piezoelectric QD and matrix.

Keywords: Quantum dots, piezoelectricity, finite element modeling, coupled thermo-piezoelectricity, Ansys.

\section{Introduction}

Quantum dots (QDs) are very small semiconductor particles, only several nanometres in size [1], so small that their optical and electronic properties differ from those of larger particles. In QDs the free carriers are confined to a small region by potential barriers in all three directions of 3D space. If the size of the region is less than the electron wavelength, the electronic states become quantized at discrete energy levels as it happens in an atom. They are a central theme in nanotechnology. Many types of QDs will emit light of specific frequencies if electricity or light is applied to them, and these frequencies can be precisely tuned by changing the dots' size, shape and material, giving rise to many applications [2 - 3]. The exploitation of this kind of nanostructures towards the improvement of the devices performance mainly relies on the ability to control their size and uniformity [4].

To bury QDs into piezoelectric matrix induces not only an elastic field, but also a piezoelectric field. The induction of both fields is given by the lattice mismatch between the QDs and the surrounding piezoelectric matrix. Since both the elastic and piezoelectric fields are equally important in the understanding of the photonic and electronic features in semiconductors [5] a reliable analysis on these fields is crucial to the design of such structures. QDs can exist in a wide variety of shapes, including cuboidal, pyramidal [6], truncated pyramidal, and lens shaped.
The stress and the strain distributions in and around the QDs have recently been investigated by many researchers [7].

The finite element method (FEM) has become an essential solution technique in many areas of engineering and physics [8]. The FEM is an efficient computational tool in the development of efficient micro and nanoscale systems [9 - 11].

In this paper a coupled 3D ANSYS Multiphysics FE model is used to simulate a cubic matrix with an embedded cubic QD under static thermal loadings. Our particular focus is on the influence of thermo-electromechanical effects on properties of QDs, where InAs (Indium arsenide) is embedded into GaAs (Gallium arsenide) matrix. The paper is organized as follows: section 2 and section 3 briefly present the governing equations for thermo-piezoelectricity and the finite element equations. Section 4 describes used simulation model in the ANSYS Multiphysics section 5 presents the numerical results. Conclusions are summarized in section 6 .

\section{Governing equations for thermo-electro-elastic fields}

The base requirement of QDs is to have initial strain, which induces electric field in a piezoelectric material. This initial strain is given by the lattice mismatch. Usually the QD has almost vanishing thermal conduction and thermal expansion

\footnotetext{
* ${ }^{1}$ Milan Zmindak, ${ }^{1}$ Pavol Novak, ${ }^{2}$ Peter Bishay

${ }^{1}$ Department of Applied Mechanics, Faculty of Mechanical Engineering, University of Zilina, Slovakia

${ }^{2}$ College of Engineering and Computer Science, California State University, Northridge, USA

E-mail: milan.zmindak@fstroj.uniza.sk
} 
coefficients. In our analysis we consider a periodic distribution of QDs in the matrix and for a numerical simulation we can select a representative volume element (RVE) illustrated in Fig. 1. This piezoelectric composite structure under a thermal load can be described by the theory of thermo- piezoelectricity.

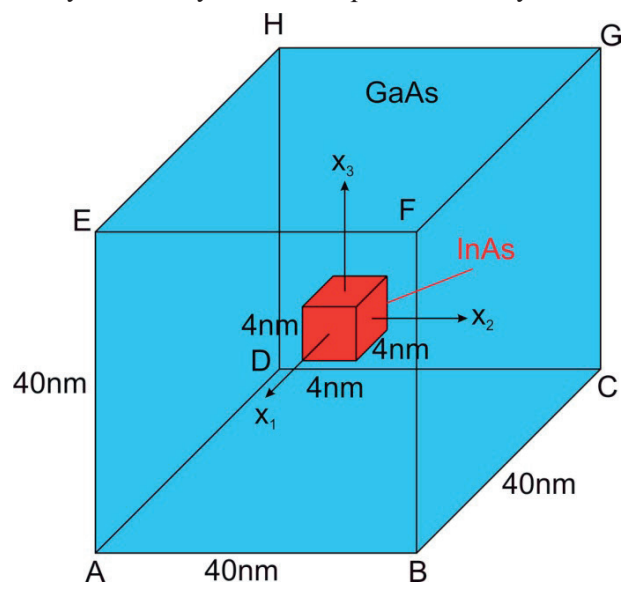

Fig. 1 Quantum dot electronic structure with InAs cubic dot in a finite sized GaAs substrate

The governing equations for thermo-piezoelectricity in a homogeneous medium are given by the balance of momentum, the first Maxwell's equation for the electric displacement vector, and heat conduction equation [12]

$\sigma_{i j, j}(\mathbf{x}, \tau)+b_{i}(\mathbf{x}, \tau)=\rho \ddot{u}_{i}(\mathbf{x}, \tau)$

$D_{j, j}(\mathbf{x}, \tau)-R(\mathbf{x}, \tau)=0$

$\psi_{i, i}(\mathbf{x}, \tau)-\rho c \dot{\theta}(\mathbf{x}, \tau)+S(\mathbf{x}, \tau)=0$,

where $\sigma_{i j}, \tau, \psi_{i}, \theta, u_{i}, D_{i}, b_{i}, R$ and $S$ are the stress tensor, time, heat flux vector, temperature difference, displacement vector, electric displacement vector, density of body force vector, volume density of free charges and density of heat sources, respectively. Also $\rho$ and $c$ are the mass density and specific heat, respectively. Dots over a quantity indicate time derivatives. A static problem can be considered formally as a special case of the dynamic one, by omitting the acceleration $\ddot{u}_{i}(\mathbf{x}, \tau)$ in the equations of motion and the time derivative term $\dot{\theta}(\mathbf{x}, \tau)$ in equation (3).

Then, the constitutive relationships express coupling of the mechanical, electrical and thermal fields and are given by

$\sigma_{i j}(\mathbf{x}, \tau)=c_{i j k l} \varepsilon_{k l}^{e}(\mathbf{x}, \tau)-e_{k i j} E_{k}(\mathbf{x}, \tau)-\gamma_{i j} \theta(\mathbf{x}, \tau)$

$D_{j}(\mathbf{x}, \tau)=e_{j k l} \boldsymbol{\varepsilon}_{k l}^{e}(\mathbf{x}, \tau)+h_{j k} E_{k}(\mathbf{x}, \tau)+p_{j} \theta(\mathbf{x}, \tau)$

where $c_{i j k l}, e_{j k l}, h_{j k}$ and $p_{j}$ are the elastic, piezoelectric, dielectric and pyroelectric material tensors in a piezoelectric medium, respectively. The stress-temperature modulus $\gamma_{i j}$ can be expressed through the stiffness coefficients and the coefficients of linear thermal expansion $\alpha_{k l}$ as

$\gamma_{i j}=c_{i j k l} \alpha_{k l}$

The thermal constitutive equation (Fourier's law) is given by

$\psi_{i}(\mathbf{x}, \tau)=\kappa_{i j} \beta_{j}(\mathbf{x}, \tau) ;$ where $\beta_{j}=\theta_{j}$

where $\kappa_{i j}$ is the thermal conductivity tensor. Both materials have transversely isotropic properties [13].

The following essential and natural boundary conditions are assumed for the mechanical field

$u_{i}(\mathbf{x}, \tau)=\bar{u}_{i}(\mathbf{x}, \tau)$, on $\Gamma_{u}$

$t_{i}(\mathbf{x}, \tau)=\sigma_{i j} n_{j}=\bar{t}_{i}(\mathbf{x}, \tau)$, on $\Gamma_{t_{i}}$

for the electric field

$\phi(\mathbf{x})=\bar{\phi}(\mathbf{x})$, on $\Gamma_{p}$,

$n_{i} D_{i}(\mathbf{x})=\bar{Q}(\mathbf{x})$, on $\Gamma_{Q}$

and for the thermal field

$\theta(\mathbf{x}, \tau)=\bar{\theta}(\mathbf{x}, \tau)$, on $\Gamma_{\theta}$

$q(\mathbf{x}, \tau)=k_{i j} \theta_{j}(\mathbf{x}, \tau) n_{i}(\mathbf{x})=\bar{q}(\mathbf{x}, \tau)$, on $\Gamma_{q}$

where $\Gamma_{u}$ is the part of the global boundary with prescribed displacements, and on $\Gamma_{t_{i}}, \Gamma_{p}, \Gamma_{Q}, \Gamma_{\theta}$, and $\Gamma_{q}$ the traction vector, the electric potential, the surface charge density, the temperature and the heat flux are prescribed, respectively.

\section{Finite element equations}

Substituting (9), (10) and (11) into (6) gives the coupled finite matrix element equation for an element

$M \ddot{q}+R \dot{q}+K q=f_{t}+f_{b}$

where

$\mathbf{M}=\iiint_{V} \mathbf{N}^{T} \rho \mathbf{N} d V ; \mathbf{R}=\iiint_{V} \mathbf{N}^{T} \lambda \mathbf{N} d V ;$

$\mathbf{K}=\iiint_{V}\left(\mathbf{B}^{T} \tilde{\mathbf{C}} \mathbf{B}+\mathbf{B}^{T} \mathbf{G N}\right) d V$

$\mathbf{f}_{\mathbf{t}}=\iint_{\Gamma_{n}} \mathbf{N}^{T}(\mathrm{~S}) \tilde{\mathbf{t}} d S ; \mathbf{f}_{\mathbf{b}}=\iiint_{V} \mathbf{w}^{T} \tilde{\mathbf{b}} d V$

where $\mathbf{N}(S)$ is the matrix of the shape functions evaluated on the boundary S. In a strongly coupled thermo-piezoelectric analysis, 
the electric potential and temperature degrees of freedom are coupled [14].

\section{Simulation model}

Using the above definitions and governing equations described in the previous section, a Finite Element Model was developed in ANSYS Multiphysics to resemble the work done in ANSYS and validate the assumptions made in the model.

The Piezoelectric Devices (PzD) user interface found under the Structural Mechanics branch in ANSYS Multiphysics, combines Solid Mechanics and Electrostatics for modeling of piezoelectric devices, for which all or some of the domains contain a piezoelectric material. The interface has the equations and features for modeling piezoelectric devices, solving for displacements and electric potential.

Conversion of material properties of piezoelectric materials (such QDs) has caused many users confusion because of the difference between manufacturer-supplied data and the format required by ANSYS. The direct method for performing a coupledfield analysis involves a single analysis using a coupled field elements SOLID226 and SOLID227. Coupled-field elements contain all the necessary degrees of freedom. They handle the field coupling by calculating the appropriate element matrices (strong, or matrix coupling) or element load vectors (weak, or load vector coupling). In linear problems with strong coupling, coupled-field interaction is calculated in one iteration. Weak coupling requires at least two iterations to achieve a coupled response. For detailed descriptions of the elements and their characteristics (DOFs, KEYOPT options, inputs and outputs, etc.), see the Element Reference [15].

\subsection{Mesh definition}

In [16], the authors performed all numerical experiments under the condition for the relative errors between successive refinements to be less than $10^{-6}$. It was achieved with around $10^{5}$ triangular elements. In our model, we define a slightly finer mesh than the default settings. The mesh is refined around the QDs to get high resolution for the calculated fields.

A 3D ANSYS model was created by constructing the geometry shown in Fig. 2 and meshing it using 195,696 SOLID elements with 339,117 nodes.

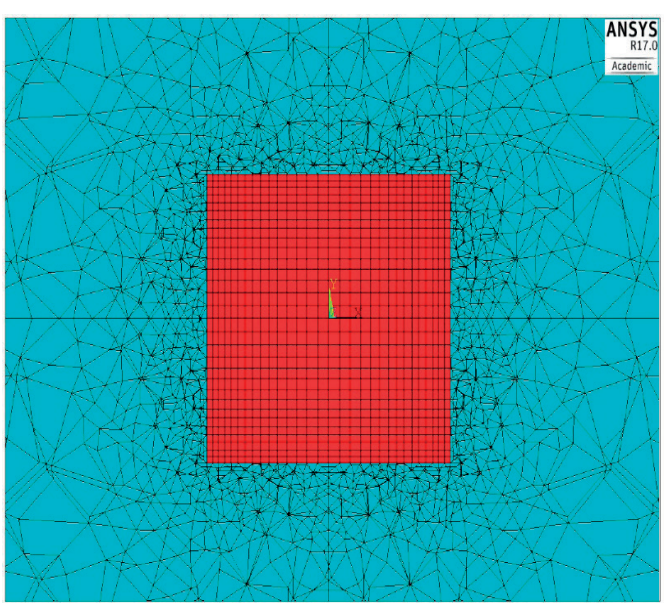

Fig. 2 Detail of FE mesh around QDS

\subsection{Boundary conditions}

The four side faces of the matrix are fixed along the direction normal to the side faces, the bottom side is fixed in all directions, top surface is free of tractions. Lateral sides and bottom side of the matrix cube are thermally isolated. Temperature is prescribed on the top side. All surfaces have vanishing normal component of electric displacement, except the bottom side, where a vanishing electric potential is prescribed. Then, one can write the following boundary conditions for the InAs/GaAs electronic structure on surfaces:

ABFE and DCGH: $u_{1}=0, t_{2}=t_{3}=0, D_{1}=0, q=0$, BCGF and ADHE: $u_{2}=0, t_{1}=t_{3}=0, D_{2}=0, q=0$, ABCD: $u_{3}=0, u_{1}=u_{2}=0, \phi=0, q=0$, EFGH: $t_{3}=0, t_{1}=t_{2}=0, D_{3}=0, \theta=\theta_{0}$.

On the interface between InAs and GaAs the continuity of displacements, electric potential and temperature has to be satisfied

$u_{i}^{m}=u_{i}^{Q D}, \phi^{m}=\phi^{Q D}, \theta^{m}=\theta^{Q D}$

as well as the reciprocity of traction vector, electric displacement and heat flux

$t_{i}^{m}+t_{i}^{Q D}=0, D_{i}^{m}+D_{i}^{Q D}=0, q^{m}+q^{Q D}=0$

In the first example stationary conditions can be considered and we consider prescribed temperature at a wide interval from 0 to $500^{\circ} \mathrm{C}$ to test behavior of the electronic microstructure. 


\subsection{Material properties}

For a periodic distribution of quantum dots in a piezoelectric matrix we consider a representative volume element (RVE) with prescribed temperature on the upper side. The RVE is assumed to have a cubic geometry with side length of $40 \mathrm{~nm}$ and a cubic quantum dot with side length of $4 \mathrm{~nm}$ is embedded in the center. Material properties of the dot correspond to InAs [17 - 19]: $c_{11}=83.3 \mathrm{GPa}, c_{12}=45.3 \mathrm{GPa}, c_{44}=39.6 \mathrm{GPa}$, $e_{14}=-0.0456, h_{11}=0.1107 \cdot 10^{-9} \mathrm{C}^{2} / \mathrm{Nm}^{2}$,

$\alpha=6.1 \cdot 10^{-6} \mathrm{deg}^{-1}, c=49.7 \mathrm{~J} / \mathrm{mol} \mathrm{deg}$,

$\kappa_{11}=46 \mathrm{~W} / \mathrm{m} \mathrm{deg}$.

Matrix (substrate) is made of GaAs, whose properties are $c_{11}=118.8 \mathrm{GPa}, c_{12}=54 \mathrm{GPa}, c_{44}=59.4 \mathrm{GPa}$, $e_{14}=-0.16, h_{11}=0.1346 \cdot 10^{-9} \mathrm{C}^{2} / \mathrm{Nm}^{2}$, $\alpha=5.1 \cdot 10^{-6} \mathrm{deg}^{-1}, c=49 \mathrm{~J} / \mathrm{mol} \mathrm{deg}$, $\kappa_{11}=0.044 \mathrm{~W} / \mathrm{m} \mathrm{deg}$.

It follows from the lattice constants that the eigenstrains are equal $\varepsilon_{11}^{*}=\varepsilon_{22}^{*}=\varepsilon_{33}^{*}=0.07$. We note that the pyroelectric coefficient $p_{1}$ is not considered in the numerical analyses as a quantity with a small influence on the results.

\section{Results and discussion}

In this example stationary conditions are considered and we consider prescribed temperature at the value $250^{\circ} \mathrm{C}$ to test behavior of the electronic microstructure. All calculations were performed for the GaAs/InAS QDs obtained from finite element analysis carried out in ANSYS Multiphysics program. The numerical results obtained by the proposed method are shown in the following figures. Figures $3 \mathrm{a}-\mathrm{c}$ and $4 \mathrm{a}-\mathrm{c}$ show the course of the electric potential and electric field in three planes. Reminded that the initial values of the mismatch induced strain of the QDs are $\varepsilon_{11}^{*}=\varepsilon_{22}^{*}=\varepsilon_{33}^{*}=0.07$. For band-gap calculations, the mismatch strain is subtracted from the actual compatible elastic strain. As a result while in the solid mechanics community the compatible elastic strain is normally expressed and plotted, the QDs research community often illustrates the subtracted strain. This can potentially cause confusion and care must be exercised in interpreting results from the solid mechanics literature. Figures 4a-c show that the electric filed component $E_{X}$ there is little temperature-dependent. Further, we can see that in inside the QDs, the magnitude of $\mathcal{E}_{x x}$, along the $X$-axis at $\mathrm{Y}=0, \mathrm{Z}=0$, decreases with an increase in temperature. The magnitude of $\boldsymbol{E}_{y y}$ along $X$-axis at $\mathrm{Y}=0, Z=0$, decreases towards zero (unstrained region) faster at higher temperature than at lower temperature. The magnitude of $\varepsilon_{z z}$ at the center of the QDs, increases with an increase in temperature.
An important point to note is that the best way to check the numerical results is to use to check the results are symmetric to $\mathrm{X}$ and $\mathrm{Y}$. For cubic materials nine planes of elastic symmetry

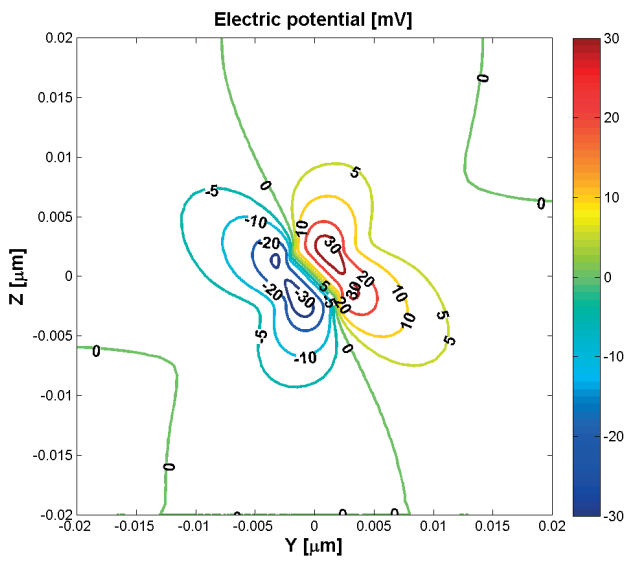

(a) plane $Y Z(X=0)$

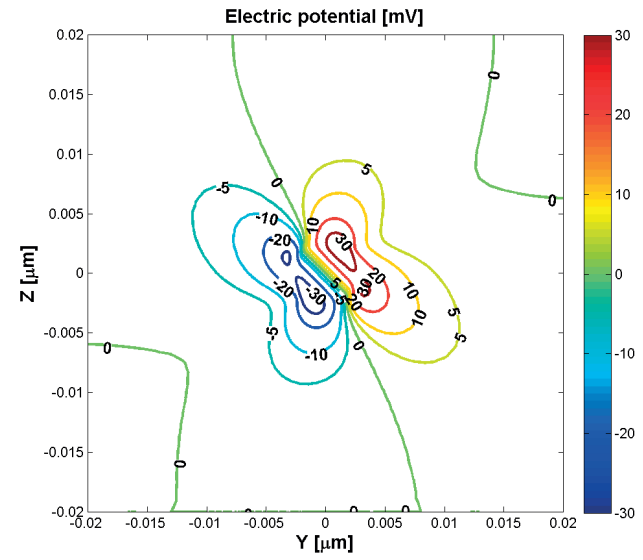

(b) plane $X Z(Y=0)$

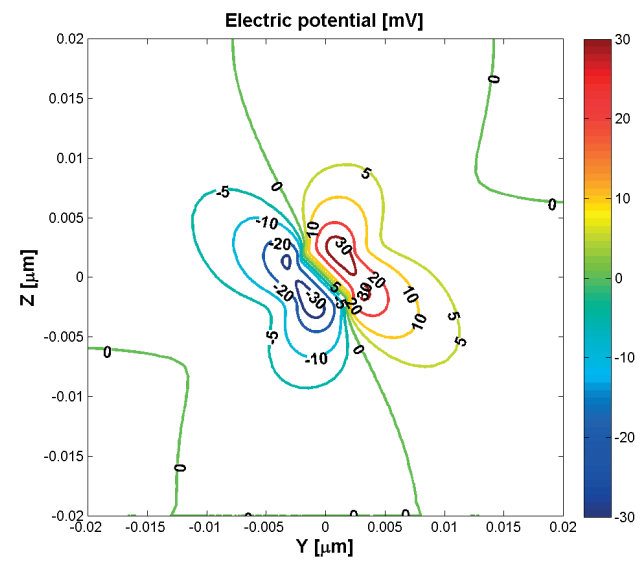

(c) plane $Y Z(X=0)$

Fig. 3 Electric potential $[\mathrm{mV}]$ in plane $Y Z(X=0)$ 
are prescribed and are derived from tetragonal symmetry [20]. Three of nine planes perpendicular to coordinate axes, are interchangeable. Six planes have normals that contain an angle

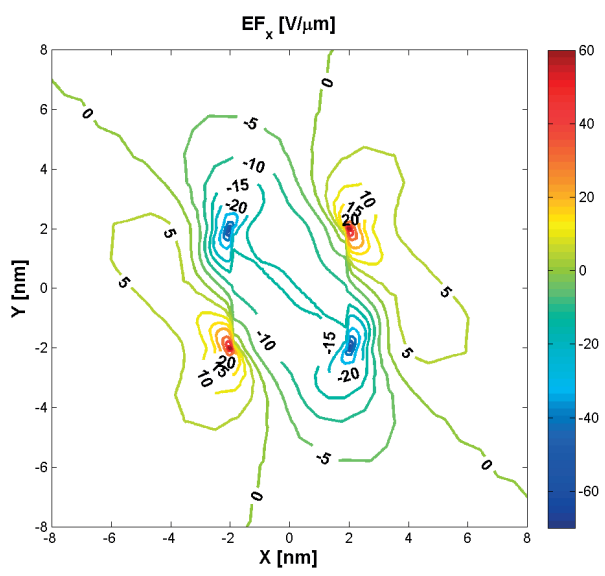

(a) plane $X Y(Z=0)$

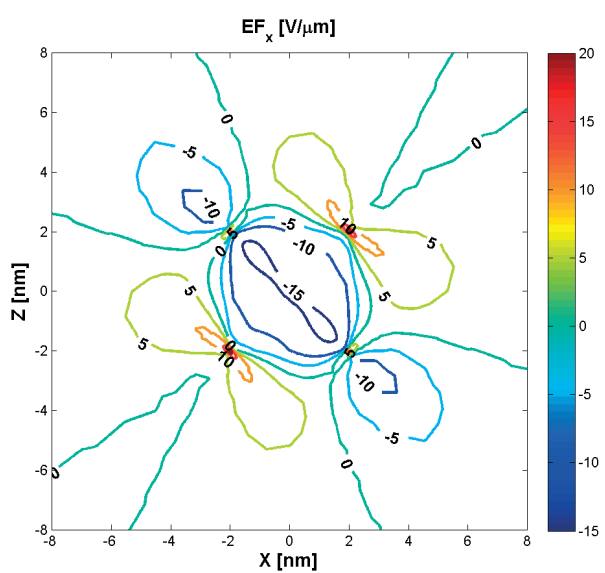

(b) plane $X Z(Y=0)$

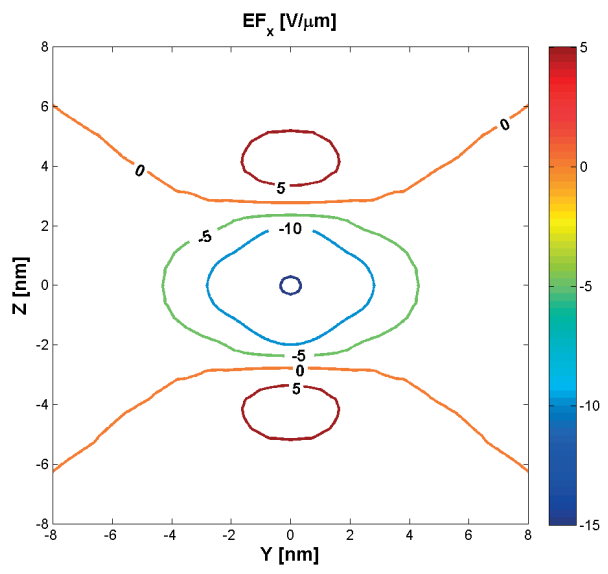

(c) plane $Y Z(X=0)$

Fig. 4 The electric field component $E_{x}[V / \mu m]$ $\pi / 4$ with the coordinate axes. Only three independent constants remain. The remaining 12 coefficients are all equal to zero. The potential difference across the top and bottom of the QD

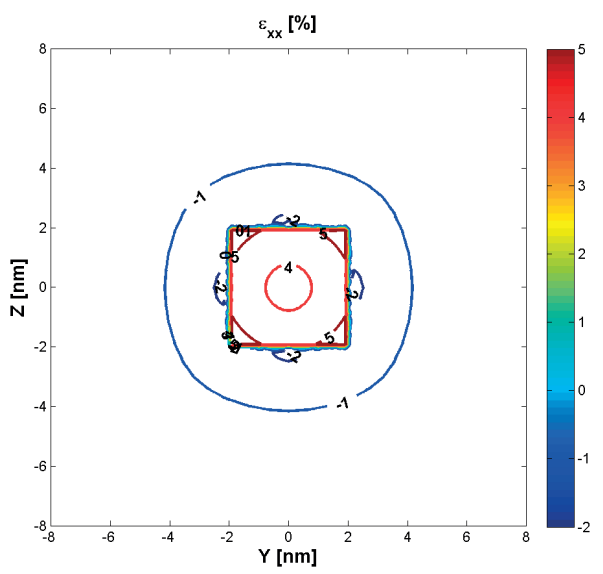

(a) $\mathcal{E}_{x x}[\%]$ in plane $Y Z(X=0)$

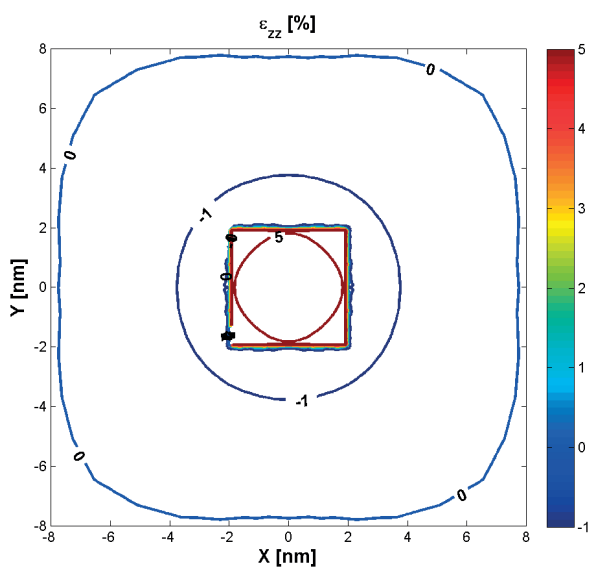

(b) $\mathcal{E}_{z z}$ in plane $X Y(Z=0)$

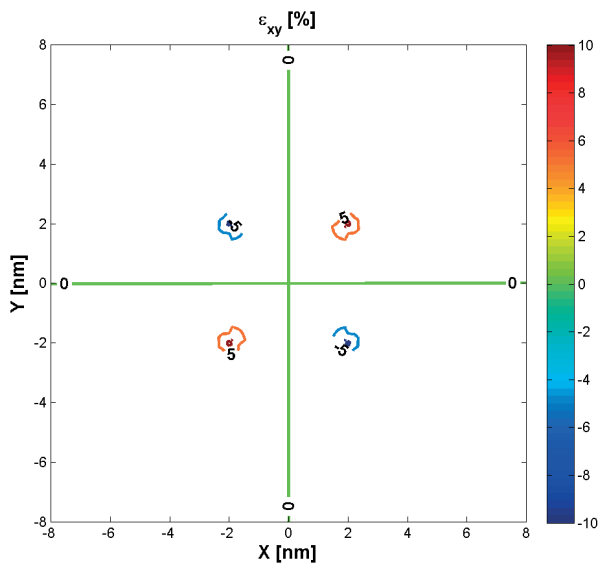

(c) $\mathcal{E}_{x y}$ in plane $X Y(Z=0)$ 


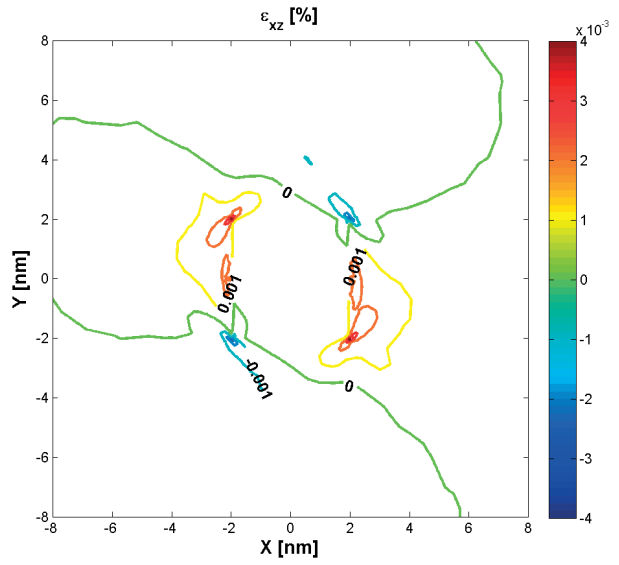

(d) $\varepsilon_{x z}$ in plane $X Y(Z=0)$

Fig. 5 The strain components $\mathcal{E}_{i j}[\%]$

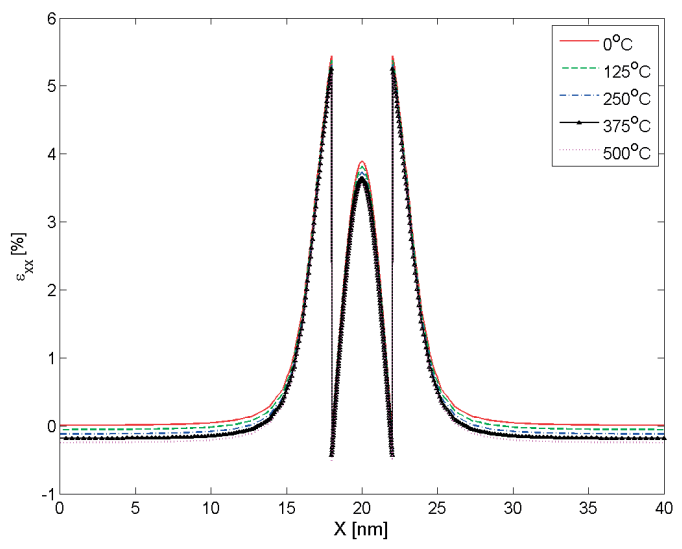

(a) The variation of strain component $\boldsymbol{E}_{x x}$ along $X$ coordinate

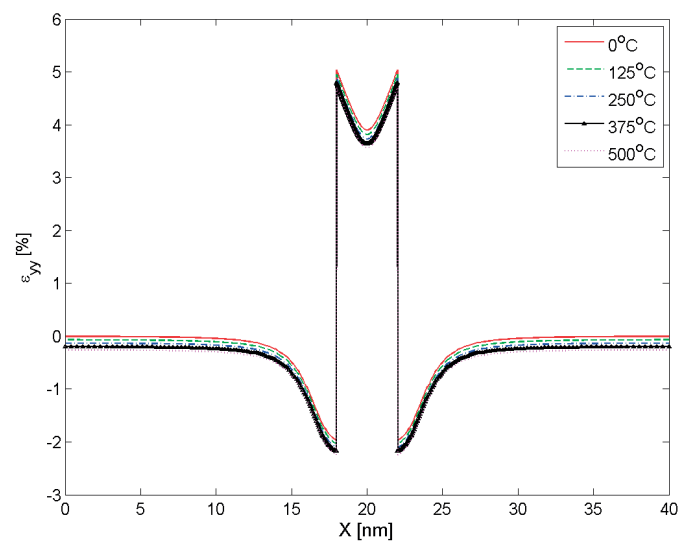

(b) The variation of strain component $\boldsymbol{\varepsilon}_{y y}$ along $X$ coordinate decreases with an increase in temperature. The electric field $E_{x}$ in plane $\mathrm{XY}$ decreases with an increase in temperature.

However, the values of electromechanical quantities show a slight shift. This shift is significant as the values for quantities like electric potential, $\mathrm{X}, \mathrm{Y}$ and Z-component of electric field vector and X-component of strain tensor, $\boldsymbol{E}_{x x}$ at the boundaries of QDs system structure.

The electric potential in planes $\mathrm{Y}=0$ and $\mathrm{Z}=0$ are similar to that in the plane $X=0$ presented in Fig. 3 due to cubic symmetry of the boundary conditions and material properties. We just need to mention that using the symmetry conditions, other $E_{i}$ on the three planes can be easily shown, with similar patterns. The strain components are presented in Figs. 5a-d.

We investigated also influence of temperature prescribed on the upper surface of the quantum dot electronic structure EFGH in Fig. 1. The above results are valid for prescribed temperature $250{ }^{\circ} \mathrm{C}$. In Figs. 6a-d there are variations of strains along $\mathrm{X}$

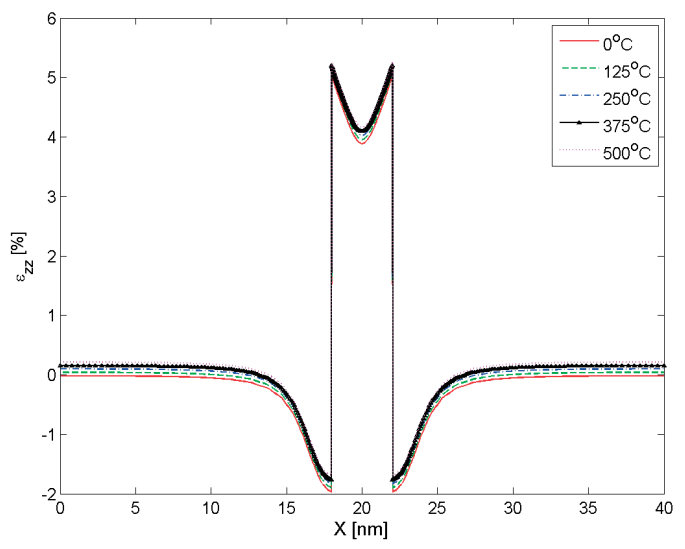

(c) The variation of strain component $\mathcal{E}_{z z}$ along $X$ coordinate

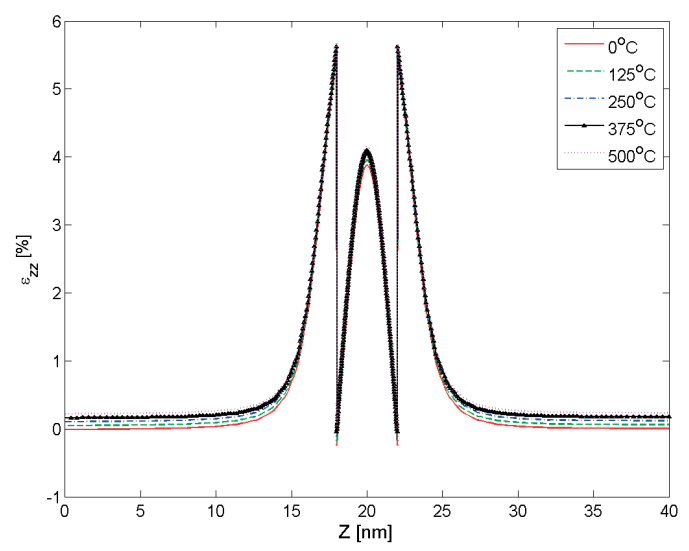

(d) The variation of strain component $\boldsymbol{\varepsilon}_{z z}$ along $Z$ coordinate

Fig. 6 The variation of strain components 
coordinate at various temperatures $125{ }^{\circ} \mathrm{C}, 250{ }^{\circ} \mathrm{C}, 375{ }^{\circ} \mathrm{C}$ and $500{ }^{\circ} \mathrm{C}$. One can observe that influence of temperature is small on values of induced strains. The induced strains are reduced in the inclusion if the temperature is enhanced. A similar conclusion can be made for variation of strains along $\mathrm{X}$ and $\mathrm{Z}$ coordinates.

\section{Conclusion}

In this paper, a three-dimensional piezoelectric FE analysis using ANSYS Multiphysics is presented to calculate the elastic and electric fields in QD nanostructures. Numerical results for the InAs/GaAs QD nanostructure show that the elastic and electric fields are strongly influenced by the differences between the material properties of the piezoelectric QD and matrix. The best way to check the numerical results is to use the results in figures and check if the results are symmetric with respect to $x$ and $y$. We are dealing with cubic materials and the results should be cubic symmetric. Once we have checked this issue, the second check is to check if the results are reasonably accurate enough by comparing these from two different mesh sizes. In this paper, we did not do this check, because the FE mesh is sufficiently fine.

\section{Acknowledgement}

The authors gratefully acknowledge support from the Slovak Grant Agency VEGA 1/0983/15.

\section{References}

[1] DAVIES J. H.: Elastic and Piezoelectric Fields around a Buried Quantum Dot: A Simple Picture. J. Appl. Phys., 84, 1998, 13581365.

[2] Available on the website: http://www.sigmaaldrich.com/materials-science/nanomaterials/ quantum-dots.html.

[3] Available on the website: https://en.wikipedia.org/wiki/Quantum_dot.

[4] ILAHI, B., SOUAF, M., BAIRA, M., ALRASHDI, J., SFAXI, L., ALHAYAA, A., MAAREL, H.: Evaluation of InAs/GaAs QQs Size with Growth Rate: A Numerical Investigation. J. of Nanomaterials, 2015, http://dx.doi.org/10.1155/2015/847018.

[5] BIMBERG, D.; GRUNDMANN, M.; LEDENTSOV N. N.: Quantum Dot Heterostructures. Wiley: New York, 1998.

[6] GRUNDMANN, M., STIER, O., BIMBERG D.: InAs/GaAs Pyramidal Quantum Dots: Strain Distribution, Optical Phonons, and Electronic Structure. Phys. Rev. B, vol. 52, 1995, 11969.

[7] ANDREEV, A. D., DOWNES, J. R., FAUX, D. A., O’ REILLY, E. P.: Strain Distribution in Quantum Dots of Arbitrary Shape. J. Appl. Phys., 86, 1999, 297-305.

[8] GASPAREC, A., SAGA, M., PECHAC, P.: Numerical Modelling of Ferrite Material Impact on the Effectiveness of Induction Heating Process. Communications - Scientific Letters of the University of Zilina, 17, 3, 2015, 12-17.

[9] MUSA, S. M.: Computational Finite Element Methods in Nanotechnology. CRC Press, 2012.

[10] BOCKO, J., LENGVARSKY, P.: Bending Vibrations of Carbon Nanotubes by using Nonlocal Theory. Procedia Engineering, 96, 2014, 21-27.

[11] MURIN, J., KUTIS, V.: An Effective Multilayered Sandwich Beam-Link Finite Element for Solution of the Electro-ThermoStructural Problems. Int. J. of Computers and Structures, 87, 2009, 1496-1507.

[12] MINDLIN, R. D.: Equations of High Frequency Vibrations of Thermopiezoelasticity Problems. Intern. J. of Solids and Structures, 10, 1974, 625-637.

[13] QIN, Q. H., YANG, Q. S.: Macro-Micro Theory on Multifield Coupling Behaviour of Heterogeneous Materials. Springer, 2008.

[14] ANSYS Mechanical APDL Coupled-Field Analysis Guide. ANSYS, Inc., 2016.

[15] ANSYS Mechanical APDL Element reference Element reference. ANSYS, Inc., 2016.

[16] PATIL, S. R., MELNIK, R. V. N.: Thermomechanical Effects in Quantum Dots. Nanotechnology, 20, 2009.

[17] GLAZOV, V. M., PASHINKIN, A. S.: Thermal Expansion and Heat Capacity of GaAs and InAs. Inorganic Materials, 2000, 225231.

[18] PAN, E., ALBRECHT, J. D., ZHANG Y.: Elastic and Piezoelectric Fields in Quantum Wire Semiconductor Structures A Boundary Integral Equation Analysis. Phys. Stat. Sol. B, 244, 2007, 1925-1939.

[19] PAN, E., CHEN, W. Q.: Static Green's Functions in Anisotropic Media. Cambridge University Press, 2015.

[20] DVORAK, G.: Micromechanics of Composite Materials. Springer, 2013. 